\title{
The role of Lactobacillus plantarum 299v in supporting treatment of selected diseases
}

\author{
KAROLINA KAŹMIERCZAK-SIEDLECKA ${ }^{1}$, AGNIESZKA DACA², MARCIN FOLWARSKI ${ }^{3}$, \\ JACEK M. WITKOWSKI ${ }^{4}$ EWA BRYL ${ }^{2}$, WOJCIECH MAKAREWICZ ${ }^{I}$
}

${ }^{1}$ Department of Surgical Oncology, Medical University of Gdansk, Gdansk, Poland

${ }^{2}$ Department of Pathology and Experimental Rheumatology, Medical University of Gdansk, Gdansk, Poland

${ }^{3}$ Department of Clinical Nutrition and Dietetics, Medical University of Gdansk, Gdansk, Poland

${ }^{4}$ Department of Physiopathology, Medical University of Gdansk, Gdansk, Poland

\begin{abstract}
Alterations in composition of human gut microbiome can lead to its dysbiosis. It is associated with gastrointestinal side effects during anti-cancer treatment, antibiotics administration, or infectious agents. There are studies confirming positive effect of consuming Lactobacillus plantarum 299v on intestinal microflora. This review summarizes the current knowledge about the role of L. plantarum $299 \mathrm{v}$ in supporting treatment of selected diseases, such as cancer, irritable bowel syndrome (IBS), and Clostridium difficile infection. The immunomodulating properties of L. plantarum $299 \mathrm{v}$ include an increase in the level of anti-inflammatory cytokines, which reduce the risk of cancer and improve the efficacy of regimens. The intake of L. plantarum 299v provides benefits for IBS patients, mainly due to normalization of stool and relief of abdominal pain, which significantly improves the quality of life of IBS patients. In addition, the intake of L. plantarum $299 \mathrm{v}$ prevents $C$. difficile-associated diarrhea among patients receiving antibiotic treatment. Due to the limited possibilities of treating these diseases and numerous complications of cancer treatment, there is a need for new therapeutic strategies. The administration of L. plantarum $299 \mathrm{v}$ seems to be useful in these cases.
\end{abstract}

Key words: Lactobacillus plantarum 299v, cancer, Clostridium difficile infection, irritable bowel syndrome, diarrhea.

(Centr Eur J Immunol 2019; 45 (4): 488-493)

\section{Introduction}

The human gut microbiome is a complex ecosystem, which consists of around 1,000 bacterial species [1], mainly from four phyla: Firmicutes, Bacteroidetes, Proteobacteria, and Actinobacteria. Usually, the most abundant of the gut microbiota are Firmicutes and Bacteroidetes [1, 2], but the exact mixture of human microbiome depend on many features, such as diet or age. Regardless of environmental factors, which can influence human microflora, the gut dysbiosis is observed if there are quantitative and qualitative changes in the composition of the gut microbiome $[1,2]$. These alterations are associated with imbalance between commensal, mutualist, and opportunist/pathobiont microorganisms [3]. It can be mainly caused by dietary habits, antibiotics administration, infectious agents, inflammatory diseases, and side effects of anti-cancer treatment $[2,3]$. As these alterations can seriously affect the effectiveness and result of ongoing treatment, which in some cases can lead to treatment termination, it is particularly crucial to supplement the imbalanced microbiome. Nowadays, there is a wide range of probiotics, which according to their definition, are 'mono- or mixed cultures of live micro-organisms which, when applied to animal or man, beneficially affect the host by improving the properties of the indigenous microflora' [4]. Amongst many proposed strains, there are many studies confirming positive effect of consuming Lactobacillus plantarum $299 \mathrm{v}$ (L. plantarum $299 v$, DMS 9843) on intestinal microflora. This review summarizes the current knowledge on the role of L. plantarum $299 \mathrm{v}$ in supporting treatment of selected diseases, such as cancer, irritable bowel syndrome, and Clostridium difficile infection.

\section{Characteristics and properties of Lactobacillus plantarum 299v}

L. plantarum $299 \mathrm{v}$ is a probiotic strain, which belongs to phylum Firmicutes and to Gram-positive lactic acid bacteria (LAB) [5]. It is commonly found in lactic acid 
of fermented food of plant origin food products, such as sauerkraut, brined olives, salted gherkins, or sourdough $[3,5,6]$. It was also proven that this particular strain is able to colonize human intestinal mucosa when orally administered [6], since it can be found in biopsy material of the mucosa in jejunum and rectum [5].

This high and still rising interest of L. plantarum 299v is due to many of its desirable features of an efficient probiotic. Among others, is its high tolerance to both low $\mathrm{pH}$ in the stomach and high $\mathrm{pH}$ as a result of the presence of bile salts in the duodenum $[3,7]$. Moreover, it allows the bacteria to transit through GI tract to the intestine, where it can influence gut microbiome. Goossens et al. in placebocontrolled double-blind study [8] evaluated the survival of L. plantarum $299 \mathrm{v}$ in both highly acidic (stomach) and alkalic (duodenum) $\mathrm{pH}$, and the impact of this strain on fecal bacterial flora. This study included 32 healthy volunteers divided into 2 groups: the first was receiving pantoprazole (proton pump inhibitor to increase stomach $\mathrm{pH}$ ) in dose $40 \mathrm{mg}$ per day, and the second group was consuming placebo for 3 weeks (from week 2 until week 4 of the study). Also, both groups were receiving $100 \mathrm{ml}$ of oatmeal fermented drink with L. plantarum $299 \mathrm{v}\left(10 \times 10^{9} \mathrm{CFU} / \mathrm{ml}\right)$, twice a day for 2 weeks (from week 3 until week 4 of the study). Fecal samples were taken at the end of week 1, 2, 4, and 8 (the $8^{\text {th }}$ week was 4 weeks after finishing pantoprazole/placebo with L. plantarum 299v supplementation). In both groups of patients, median count of lactobacilli (identified as L. plantarum 299v) increased significantly. In pantoprazole group in the fourth week compared to the first week of the study, the level increased from $\log 4.5$ to 8.0 CFU/g feces and decreased significantly in the eighth week (to $\log 4.5 \mathrm{CFU} / \mathrm{g}$ feces). In placebo group, median count increased from $\log 4.2$ to $7.7 \mathrm{CFU} / \mathrm{g}$ feces and then decreased to $4.3 \mathrm{CFU} / \mathrm{g}$ feces. No significant alterations in amounts of other bacteria (both aerobic, e.g. Escherichia coli, and anaerobic, e.g. Clostridia spp. and Bacteroides spp.) and concentrations of short-chain fatty acids (SCFA) were observed. The lack of increase in SCFA concentration might relate to the fact that they were measured in feces, where the concentration of SCFA is just 5\%, and the rest is absorbed in the colon. Overall, these results confirm that L. plantarum $299 \mathrm{v}$ has the ability to survive passage through gastrointestinal tract, including acid conditions of the human stomach [8].

L. plantarum 299v can also affect the survival of other pathogenic bacteria regardless of its use in mixtures or in formulations with a single strain. Johansson et al. showed that after consuming mixture of lactic acid bacteria (including L. plantarum 299v), the levels of LAB in human feces and jejunum mucosa increased. On the other hand, the amount of sulphite-reducing clostridia, anaerobic gram-positive bacteria as well as gram-negative anaerobic bacteria of the rectal mucosa, significantly decreased after administration of L. plantarum 299v formula [7].
L. plantarum $299 \mathrm{v}$ can influence innate immunity of gut-associated lymphoid tissue (GALT). It increases both the transcription of genes encoding mucins (MUC2 and MUC3) and their excretion in goblet cells [9, 10]. Mucins are glycoproteins, which provide protection to intestinal mucosal surfaces. In that way, they can interrupt colonization and invasion of pathogens, and by influencing viral replication, they can efficiently limit viral disease [9]. For this reason (by stimulation of mucins production and release), L. plantarum $299 \mathrm{v}$ can indirectly inhibit the adhesion of enteropathogenic Escherichia coli to intestinal epithelial cells [10].

The repressive activity of $L$. plantarum $299 \mathrm{v}$ is also associated with a production of inhibitory compounds, such as organic acids. Johansson et al. showed that administration of L. plantarum 299v (in dose of $5 \times 10^{7} \mathrm{CFU} \mathrm{ml}^{-1}$ for 21 days in healthy volunteers) significantly increased the total faecal concentration of acetic and propionic acids [11], which as both being SCFA, they can affect local $\mathrm{pH}$ of intestine. By lowering $\mathrm{pH}$, they control proliferation of pathogens and by influencing the blood flow through the intestinal vessels, they affect e.g. colonic- hepatic portal venous blood flow [12]. In Johansson et al. study, as the effect of organic acid production, a decrease of the amount of sulphite-reducing clostridia was observed, while the numbers of total anaerobes, gram-negative anaerobes, and total aerobes did not change [11]. That suggest that L. plantarum $299 \mathrm{v}$ can affect metabolic activity of other bacteria in colon (L. plantarum 299v cannot produce acetic or propionic acids). It can also inhibit the growth of potentially pathogenic bacteria such as Listeria monocytogenes, Bacillus cereus, Yersinia enterocolitica, Citrobacter freundii, Enterobacter cloacae, and Enterococcus faecalis $[11,13]$.

The ability of L. plantarum $299 \mathrm{v}$ to adhere to the intestinal wall also classifies the strain as a good probiotic, since is it able to reside in human mucosal cells in vivo [9]. The adhesion is based on the mechanism of mannose-binding [7, 9], which is essential for immunomodulating properties of this strain. Mao et al. examined the gut immune response to oral administration of $L$. plantarum $299 \mathrm{v}$, Lactobacillus reuteri $R 2 L C$, pectin, and oat base on methotrexate-induced enterocolitis in rats. The animal models were divided into 2 groups, where the treatment group received elemental diet with pectin, oat base, and probiotics (L. plantarum $299 \mathrm{v}$ and $L$. reuteri $R 2 L C$ ) via a gastrostomy, and the control group had normal diet. On $3^{\text {rd }}$ day of the study, rats received methotrexate in dose of $20 \mathrm{mg} / \mathrm{kg}$ or normal saline. The blood samples were taken on day 6 , and the level of intestinal secretory $\operatorname{Ig} \mathrm{A}(\operatorname{sg} \mathrm{A})$, the count of gut lamina propria CD4+ (T helper lymphocytes, Th), and CD8+ (T cytotoxic lymphocytes, Tc) lymphocytes were significantly diminished by administration of methotrexate. After consuming probiotics (not pectin) and oat base only, the level of ileal and colonic sIgA sig- 
nificantly increased. Additionally, the count of gut lamina propria CD4+ and CD8+ lymphocytes normalized [14]. Rask et al. evaluated the influence of five different strains of Lactobacilli (L. plantarum 299v, L. plantarum HEAL, L. rhamnosus, L. gasseri, L. fermentum, and L. paracasei), or Pseudomonas lundensis or placebo in human immune system condition by administering lyophilized bacteria, with $10^{9}$ /day for $P$. lundensis and $10^{10}$ day for Lactobacilli (or placebo), for 2 weeks. The blood was taken on the day before starting the supplementation, on the $14^{\text {th }}$ day (for all groups), and in $5^{\text {th }}$ week (for the group taking placebo and L. plantarum $299 \mathrm{v}$ ) of supplementation. The results of the tests showed that changes in acquired immune system cells were most evident in case of L. plantarum $299 \mathrm{v}$ supplementation. The strain was able to most notably increase the expression of activation markers on the surface of CD4+ and CD8+ T lymphocytes, suggesting that the ingestion of bacteria leads to the activation of APC (antigen presenting cells) and T cells, or general increase of acquired immunity. The effect on innate immunity, measured as the ability to produce IL-12 by monocytes/macrophages, was not as evident [15].

\section{Use of Lactobacillus plantarum 299v in oncology}

It is a well-known fact that the immune system plays an important role in the prevention and control of tumor initiation and progression [16]. Lactic acid bacteria have the ability to modulate the immune system [3, 17]. As mentioned above, Rask et al. study demonstrated that an intake of $L$. plantarum $299 \mathrm{v}$ increases the expression of activation markers in CD8+ T cells, and the memory cells marker's (CD45RO) presence in CD4+ T cells also increases [15]. As Rask et al. suggested, it is hard to believe that the evident changes observed in the level of activating markers on the surface of CD4+ and CD8+ T lymphocytes are the direct result of $\mathrm{T}$ lymphocytes stimulation by L. plantarum $299 \mathrm{v}$; it is rather the effect on antigen presenting cells (APC). Irrespective of the reason, it is safe to assume that $L$. plantarum $299 \mathrm{v}$ may enhance the immune defense against viral infections and tumors.

L. plantarum 299v may also be useful in reducing mucosal inflammation and in stimulating intestinal mucosal growth. In their study, Liu et al. [18] examined Sprague-Dawley rats, which received external radiation of the lower abdomen and underwent colonic resection with anastomosis. It was observed that the group of rats receiving L. plantarum $299 \mathrm{v}$ was characterized by higher concentration of collagen content in anastomotic parts, which reflected better capacity for wound healing, as compared with the control group receiving saline. The MPO (tissue myeloperoxidase) activity indicating leukocytes accumulation and inflammatory response was significantly decreased in the L. plantarum $299 \mathrm{v}$ group compared to the control group, which is beneficial for healing of the wound and radiation injury. It can be concluded that administration of L. plantarum $299 \mathrm{v}$ reduces the intestinal injury and inflammation after external radiation. Moreover, it improves colonic anastomotic healing.

The gut-associated lymphoid tissue is one of the most important part of gut barrier. Animal studies showed that L. plantarum $299 \mathrm{v}$ improves the immunological status of intestinal mucosa. However, it was not proven in Woodcock et al. trial [19] with surgical patients (colorectal carcinoma $n=12$, Crohn's disease $n=4$, ulcerative colitis $n=2$, gastric carcinoma $n=1$, colonic adenoma $n=1$, angiodysplasia $n=1$, enterocutaneous fistula $n=1$ ) divided into 2 groups (probiotic $n=11$, control $n=11$ ). The influence of L. plantarum $299 \mathrm{v}$ probiotic on the condition of GALT was assessed by verifying the changes in concentrations of plasma cells, IgA positive cells, and IgM positive cells in the lamina propria. The concentration of $\operatorname{IgA}$ and $\operatorname{IgM}$ antibodies on the mucosal surface was also examined. The only difference observed between those two groups was higher level of IgM antibodies on the mucosal surface in the control group. Other parameters did not differ in both groups challenging the results of other experiments claiming immunomodulatory effect of L. plantarum 299v. Lack of evident positive effect of L. plantarum $299 \mathrm{v}$ supplementation in those patients could be the result of heterogeneity of tested groups regarding both primary disease and peripheral immune system and GALT conditions, which were not assessed before starting the supplementation. Other study [20] examined if administration of $L$. plantarum $299 \mathrm{v}$ modulates the gut barrier function in patients with obstructive jaundice (12 patients with choledocholithiasis, 9 with a periampullary tumors, and 4 other conditions), of which $1 / 3$ of patients received L. plantarum $299 \mathrm{v}, 1 / 3$ of patients received placebo, and $1 / 3$ of patients received water. The intestinal permeability was measured by following parameters: concentration of tumor necrosis factor (TNF) p55 receptor, which reflects an acute inflammatory response of the immune system, and lactulose/mannitol (L/M) ratio corresponding to intestinal permeability. These parameters were measured the day before biliary drainage and on days 1 and 7 after biliary drainage. At the beginning, the median L/M ratio was 0.035 (range, 0.018-0.065), and there was no difference between groups before drainage and on $7^{\text {th }}$ day after drainage. On the first day after biliary drainage, the L/M ratio was lower in group receiving L. plantarum $299 \mathrm{v}$ compared with the other group, but the difference was not statistically significant. Also, the TNF p55 receptor level $(15.3 \mathrm{ng} / \mathrm{ml} ; p=0.43)$ was lower in the group receiving L. plantarum $299 \mathrm{v}$ on day 1 after biliary drainage compared with placebo $(30.9 \mathrm{ng} / \mathrm{ml})$ and the group receiving water $(82.7 \mathrm{ng} / \mathrm{ml})$, but as in case of L/M ratio, the difference was not statistically significant. These trends suggest that administration of L. plantarum 299v improves 
intestinal permeability after biliary drainage and attenuates the inflammatory response, but there is a need for further evaluation in a larger group of patients.

To conclude, presented trials suggest that intake of L. plantarum 299v may enhance immunological status of the intestinal mucosa. However, additional and larger multicenter studies are necessary to confirm the effect on clinical outcomes.

\section{Impact of Lactobacillus plantarum 299v on Clostridium difficile infections treatment}

C. difficile infections (CDI) are one of the most common cause of diarrhea among patients receiving antibiotics. The death rate amongst those patients is quite high. There are studies, which confirm the potential role of L. plantarum 299v in the prevention of CDI. KujawaSzewieczek et al. [5] examined the effect of routine use of L. plantarum $299 \mathrm{v}$ as a prevention of CDI. This study included 3,533 patients hospitalized in the nephrology and transplantation ward, during a two-year period. From October 2012 to October 2013, patients treated with antibiotics received probiotics, such as Saccharomyces boulardii, Lactobacillus rhamnosus, Lactobacillus acidophilus, Lactobacillus delbrueckii, and Bifidobacterium lactis. From December 2013 to December 2014, all patients consumed one capsule of Sanprobi IBS ${ }^{\circledR}\left(10 \times 10^{9}\right.$ colony-forming units (CFU) of L. plantarum 299v) daily. The patients who received $L$. plantarum $299 \mathrm{v}$ have shown a significant decrease of the incidence of CDI. This study suggests that the use of this probiotic strains during treatment with antibiotics may prevent CDI. The same authors published a paper 3 years later, in which confirmed that the routine use of L. plantarum 299v may prevent CDI during antibiotic therapy in patients who received immunosuppressive agents and remained in nephrology and transplantation ward [21]. Another study [22] evaluated the role of L. plantarum 299v in the treatment of CDI in critically ill patients in intensive care unit (ICU). A total of 22 patients received a fermented oatmeal gruel with L. plantarum $299 \mathrm{v}\left(10 \times 10^{8}\right.$ colony-forming units (CFU)/ml of L. plantarum $299 \mathrm{v}$ ), and 22 patients consumed the same product, but without the bacteria (control group). The product was given as bolus doses at the beginning in six $100 \mathrm{ml}$ doses of gruel at 12 hours intervals and thereafter, $50 \mathrm{ml}$ doses two times a day, until the patient stayed in the ICU. Fecal samples were taken at inclusion and then twice a week during ICU stay. Participants receiving L. plantarum 299v had a negative $C$. difficile test. Four patients (19\%) from the control group had a positive $C$. difficile test. This study suggests that $L$. plantarum $299 \mathrm{v}$ counteracts or even prevents the colonization with $C$. difficile in critically ill patients treated with antibiotics. Additionally, there was an improvement in lactulose/rhamnose ratio in the group receiving L. plantarum $299 \mathrm{v}$, which again confirms the influence of
L. plantarum $299 \mathrm{v}$ on intestinal permeability. In general, due to the small number of participants in the above mentioned study, there is a need for further studies to confirm these results [22].

Short-chain fatty acids, which belong to carboxylic acids have many advantageous properties. Apart from being an energy source for the mucosal cells in the colon (butyrate), they may inhibit growth of certain microbes [23], stimulate colonic epithelial proliferation [12], and stimulate colonic electrolyte transport improving diarrheal symptoms [12]. Also, an increased concentration of shortchain fatty acids has a positive effects on intestinal microflora [6, 11]. In their study, Wullt et al. [24] investigated how the administration of L. plantarum $299 \mathrm{v}$ enhances the concentrations of fecal organic acids after metronidazole treatment. This trial included 19 patients with recurrent C. difficile-associated diarrhea. After a ten days intake of metronidazole, the total amount of short-chain fatty acids significantly decreased in the placebo group, whereas in the L. plantarum 299v group, such trend was not observed (decrease from 77.1 to $45.5 \mu \mathrm{mol} / \mathrm{g}$ in placebo group, $p=0.028$; and from 79.8 to $60.4 \mu \mathrm{mol} / \mathrm{g}$ in L. plantarum $299 v$ group). Statistically significant difference was observed between groups in butyrate production: in placebo group, it decreased from 5.6 to $1.2 \mu \mathrm{mol} / \mathrm{g}$ after 10 days of metronidazole treatment and in L. plantarum $299 \mathrm{v}$ group, it decreased from 7.6 to $5.6 \mu \mathrm{mol} / \mathrm{g}$. The concentration of succinate significantly decreased in both treatment groups (placebo group: from 6.3 to $1.5 \mu \mathrm{mol} / \mathrm{g}$; L. plantarum 299v group: from 9.3 to $0.9 \mu \mathrm{mol} / \mathrm{g}, p=0.028$ ). This study showed that the intake of L. plantarum $299 \mathrm{v}$ reduced negative effects of antibiotics in colonic fermentation, and could provide additional benefits for patients suffering from recurrent $C$. difficile-associated diarrhea [24].

\section{Advantages of using Lactobacillus plantarum 299v in irritable bowel syndrome treatment}

Irritable bowel syndrome (IBS) is a functional gastrointestinal disorder of unknown etiology [25]. The most common symptoms of IBS are abdominal pain, flatulence, and irregular bowel movement $[4,25,26]$. One of the factors contributing to increasing IBS symptoms are alterations in intestinal bacteria microflora [25]. Results of study performed on 52 patients with IBS fulfilling Rome criteria showed that the intake of L. plantarum 299v (rosehip drink containing $5 \times 10^{7} \mathrm{CFU} / \mathrm{ml}$ of L. plantarum $299 \mathrm{v}$, in dose of $400 \mathrm{ml}$ per day, for 4 weeks) reduced abdominal pain and flatulence [25]. Similar results were obtained in a study by Ducrotte et al. [4], where the following symptoms were assessed: decrease of the frequency of abdominal pain episodes, changes in severity of abdominal pain, changes in frequency and severity of abdominal bloating, 
and feeling of incomplete rectal emptying. IBS patients ( $n=214$ ) fulfilling the Rome III criteria were divided into two groups: test group received L. plantarum $299 \mathrm{~V}$ $(n=108)$ and placebo group $(n=106)$. The test product was a capsule in a potato starch and magnesium stearate base, which contained $10 \times 10^{9} \mathrm{CFU}$ of L. plantarum $299 \mathrm{v}$ per dose. The control product had a similar appearance, texture, and taste, but did not contain probiotic. Participants received one capsule of L. plantarum 299v or placebo per day for four weeks. The abdominal pain severity and daily frequency were significantly lower in the test group compared with the placebo group $(p<0.05)$ as early as in the $2^{\text {nd }}$ week (out of 4 ) of probiotic consumption. $78.1 \%$ of participants declared that symptomatic effect was excellent or good after consuming L. plantarum 299v, while only $8.1 \%$ of participants from the placebo group claimed the same effect [4]. Another study [27] also examined the efficacy of $L$. plantarum $299 \mathrm{v}$ in the treatment of IBS. This study included 40 patients divided into two groups: receiving L. plantarum $299 \mathrm{v}$ in liquid suspension for 4 weeks $\left(5 \times 10^{7} \mathrm{CFU} / \mathrm{ml}\right.$, in dose of $200 \mathrm{ml}$, twice a day) $(n=20)$ and placebo $(n=20)$. The patients assessed their IBS symptoms applying a scoring system. Clinical test was performed at the beginning and after the study. The resolution of abdominal pain was declared by all patients receiving $L$. plantarum $299 \mathrm{v}$ and by only 11 patients in the group consuming placebo $(p=0.0012)$. The normalization of stool frequency was observed in 6 out of 10 patients receiving L. plantarum 299v comparing with 2 out of 11 patients from the placebo group ( $p=0.17)$. The improvement of all IBS symptoms was noted in $95 \%$ patients from experimental group, but only in $15 \%$ from placebo group $(p<0.0001)$ [27]. In summary, the above-mentioned studies confirm that administration of L. plantarum $299 \mathrm{v}$ seems to be useful in improving the symptoms of IBS. Additionally, it is worth to consider L. plantarum 299v formulations as valuable co-treatment of IBS, especially after conducting further studies with larger cohorts.

\section{Conclusions}

L. plantarum $299 \mathrm{v}$ is a probiotic strain of lactic acid bacteria naturally appearing in human gut, which can modulate the immune system. Its immunomodulating properties are observed among others in decreasing the level of anti-inflammatory cytokines. The intake of L. plantarum $299 \mathrm{v}$ reduces IBS symptoms and improves the quality of life of these patients. The administration of L. plantarum $299 \mathrm{v}$ is also useful in treatment of $C$. difficile-associated diarrhea. Further studies should focus on the use of L. plantarum $299 \mathrm{v}$ in supporting cancer treatment, in gastrointestinal reduction of side effects, and an improvement of quality of life.

The results obtained from various studies show incontestable positive influence of L. plantarum $299 \mathrm{v}$ on human microbiome and overall body functioning, even in patients with serious health problems. This positive effect seems to withstand huge diversity of cohorts taking part in presented trials. Age and sex differences, size of populations observed, and their various origin did not affect the final positive effect of L. plantarum 299v on overall human health.

The lack of side effects after taking L. plantarum $299 \mathrm{v}$, even in critically ill patients, increases the value of this strain even further as a probiotic, because it underlines its non-invasive character.

The authors declare no conflict of interest.

\section{References}

1. D'Argenio V, Salvatore F (2015): The role of the gut microbiome in the healthy adult status. Clin Chim Acta 451 (Part A): 97-102.

2. Sun J, Chang EB (2014): Exploring gut microbes in human health and disease: Pushing the envelope. Genes Dis 1: 132139.

3. Banna GL, Torino F, Marletta F, et al. (2017): Lactobacillus rhamnosus GG: an overview to explore the rationale of its use in cancer. Front Pharmacol 8: 603.

4. Ducrotté P, Sawant P, Jayanthi V (2012): Clinical trial: Lactobacillus plantarum 299v (DSM 9843) improves symptoms of irritable bowel syndrome. World J Gastroenterol 18: 40124018.

5. Kujawa-Szewieczek A, Adamczak M, Kwiecień K, et al. (2015): The effect of Lactobacillus plantarum 299v on the incidence of Clostridium difficile infection in high risk patients treated with antibiotics. Nutrients 7: 10179-10188.

6. Molin G (2001): Probiotics in foods not containing milk or milk constituents, with special reference to Lactobacillus plantarum 299v. Am J Clin Nutr 73: 380-385.

7. Johansson ML, Molin G, Jeppsson B, et al. (1993): Administration of different Lactobacillus strains in fermented oatmeal soup: in vivo colonization of human intestinal mucosa and effect on the indigenous flora. Appl Environ Microbiol 59: 15-20.

8. Goossens D, Jonkers D, Russel M, et al. (2005): Survival of the probiotic, L. plantarum $299 \mathrm{v}$ and its effects on the faecal bacterial flora, with and without gastric acid inhibition. Dig Liver Dis Off J Ital Soc Gastroenterol Ital Assoc Study Liver 37: 44-50.

9. Mack DR, Ahrne S, Hyde L, et al. (2003): Extracellular MUC3 mucin secretion follows adherence of Lactobacillus strains to intestinal epithelial cells in vitro. Gut 6: 827-833.

10. Mack DR, Michail S, Wei S, et al. (1999): Probiotics inhibit enteropathogenic E. coli adherence in vitro by inducing intestinal mucin gene expression. Am J Physiol 52: 941-950.

11. Johansson M-L, Nobaek S, Berggren A, et al. (1998): Survival of Lactobacillus plantarum DSM $9843(299 \mathrm{v})$, and effect on the short-chain fatty acid content of faeces after ingestion of a rose-hip drink with fermented oats. Int J Food Microbiol 42: 29-38.

12. Topping DL (1996): Short-chain fatty acids produced by intestinal bacteria. Asia Pac J Clin Nutr 5: 15-19.

13. Jacobsen CN, Rosenfeldt Nielsen V, Hayford AE, et al. (1999): Screening of probiotic activities of forty-seven strains 
of Lactobacillus spp. by in vitro techniques and evaluation of the colonization ability of five selected strains in humans. Appl Environ Microbiol 65: 4949-4956.

14. Mao Y, Yu J-L, Ljungh A, et al. (1996): Intestinal Immune Response to Oral Administration of Lactobacillus reuteri R2LC, Lactobacillus plantarum DSM 9843, Pectin and Oatbase on Methotrexate-induced Enterocolitis in Rats: Microbial Ecology in Health and Disease 9: 261-269.

15. Rask C, Adlerberth I, Berggren A, et al. (2013): Differential effect on cell-mediated immunity in human volunteers after intake of different lactobacilli. Clin Exp Immunol 172: 321-332.

16. Dasari S, Kathera C, Janardhan A, et al. (2017): Surfacing role of probiotics in cancer prophylaxis and therapy: a systematic review. Clin Nutr Edinb Scotl 36: 1465-1472.

17. Ferreira dos Santos T, Alves Melo T, Almeida ME, et al. (2016): Immunomodulatory effects of Lactobacillus plantarum Lp62 on intestinal epithelial and mononuclear cells. Biomed Res Int 2016: 8404156.

18. Liu Q, Nobaek S, Adawi D, et al. (2001): Administration of Lactobacillus plantarum 299v reduces side-effects of external radiation on colon anastomotic healing in an experimental model. Colorectal Dis Off J Assoc Coloproctology G B Irel 3: 245-252.

19. Woodcock NP, McNaught CE, Morgan DR, et al. (2004): An investigation into the effect of a probiotic on gut immune function in surgical patients. Clin Nutr Edinb Scotl 23: 1069-1073.

20. Jones C, Badger SA, Regan M, et al. (2013): Modulation of gut barrier function in patients with obstructive jaundice using probiotic LP299v. Eur J Gastroenterol Hepatol 25: 1424-1430.

21. Dudzisz S, Kujawa-Szewieczek A, Kwiecień K, et al. (2018): Lactobacillus plantarum 299v reduces the incidence of Clostridium difficile infection in nephrology and transplantation ward- results of one year extended study. Nutrients 10: 1574.

22. Klarin B, Wullt M, Palmquist I, et al. (2008): Lactobacillus plantarum 299v reduces colonisation of Clostridium difficile in critically ill patients treated with antibiotics. Acta Anaesthesiol Scand 52: 1096-1102.

23. Mortensen FV, Mřller JK, Hessov I (1999): Effects of shortchain fatty acids on in vitro bacterial growth of Bacteroides fragilis and Escherichia coli. APMIS Acta Pathol Microbiol Immunol Scand 2: 240-244.

24. Wullt M, Johansson Hagslätt M-L, Odenholt I, Berggren A (2007): Lactobacillus plantarum $299 \mathrm{v}$ enhances the concentrations of fecal short-chain fatty acids in patients with recurrent clostridium difficile-associated diarrhea. Dig Dis Sci 52: 2082-2086.

25. Nobaek S, Johansson ML, Molin G, et al. (2000): Alteration of intestinal microflora is associated with reduction in abdominal bloating and pain in patients with irritable bowel syndrome. Am J Gastroenterol 95: 1231-1238.

26. Marlicz W, Zawada I, Starzyńska T (2012): Irritable bowel syndrome--irritable bowel or irritable mind? Pol Merkur Lek Organ Pol Tow Lek 32: 64-69.

27. Niedzielin K, Kordecki H, Birkenfeld B (2001): A controlled, double-blind, randomized study on the efficacy of Lactobacillus plantarum $299 \mathrm{~V}$ in patients with irritable bowel syndrome. Eur J Gastroenterol Hepatol 13: 1143-1147. 